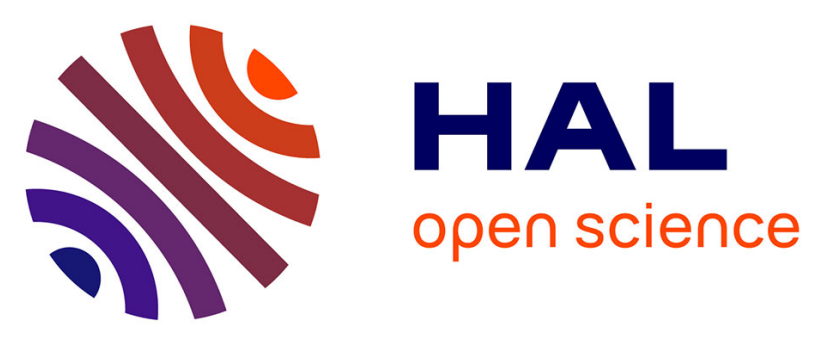

\title{
Nominal GNSS pseudorange measurement model for vehicular urban applications
}

\author{
Daniel Salós, Christophe Macabiau, Anaïs Martineau, Bernard Bonhoure, \\ Damien Kubrak
}

\section{- To cite this version:}

Daniel Salós, Christophe Macabiau, Anaïs Martineau, Bernard Bonhoure, Damien Kubrak. Nominal GNSS pseudorange measurement model for vehicular urban applications. IEEE/ION PLANS 2010, Position Location and Navigation Symposium, May 2010, Indian Wells, United States. pp 806-815, 10.1109/PLANS.2010.5507319 . hal-01022507

\section{HAL Id: hal-01022507 https://hal-enac.archives-ouvertes.fr/hal-01022507}

Submitted on 30 Sep 2014

HAL is a multi-disciplinary open access archive for the deposit and dissemination of scientific research documents, whether they are published or not. The documents may come from teaching and research institutions in France or abroad, or from public or private research centers.
L'archive ouverte pluridisciplinaire HAL, est destinée au dépôt et à la diffusion de documents scientifiques de niveau recherche, publiés ou non, émanant des établissements d'enseignement et de recherche français ou étrangers, des laboratoires publics ou privés. 


\section{Nominal GNSS Pseudorange Measurement Model for Vehicular Urban Applications}

\author{
Daniel Salós \\ TéSA/ENAC \\ Toulouse, France
}

\author{
Christophe Macabiau, Anaïs Martineau \\ ENAC \\ Toulouse, France
}

\author{
Bernard Bonhoure \\ CNES \\ Toulouse, France
}

\author{
Damien Kubrak \\ Thales Alenia Space \\ Toulouse, France
}

\begin{abstract}
Certain GNSS applications conceived for road users in urban scenarios must meet some particular integrity requirements to assure the system safety, reliability or credibility. For instance, GNSS-based Road User Charging is one of these applications that recently has attracted special interest. A correct design of such applications needs the knowledge of the GNSS error distribution. Furthermore, the GNSS error model should have been built with overbounding techniques. The user is a vehicle equipped with a GNSS receiver that may track different signals of various systems (GPS, Galileo, SBAS), in a single- or dual-frequency configuration. The different error sources contributing to the total pseudorange error are identified, analyzed and modeled, using overbounding techniques when necessary. Finally the pseudorange measurement error model is obtained and analyzed for different receiver configurations.
\end{abstract}

Keywords- Nominal Measurement Error; GNSS Integrity; Road and Urban Environments

\section{INTRODUCTION}

Safety- and liability-critical GNSS applications are defined by the fact that large and undetected position errors may have negative safety, legal or economic implications. Many of them are services conceived for vehicles circulating in urban and suburban areas, such as management of hazardous goods transport or road user charging (RUC) systems among others. In particular, GNSS-based electronic toll collection (ETC) systems have recently attracted special attention due to their flexibility and potential reduction of side-road infrastructures. GNSS integrity control plays a main role in these applications quantifying the trust of the information provided by the GNSS and maintaining under control the negative effects derived from large undetected position errors. In other words, integrity control is a key parameter to assure the system reliability and credibility.

An appropriate error characterization is fundamental to design integrity-based applications and to foresee their performances. In particular, large errors that threat the integrity of the system must be considered in the error model, even if they rarely occur, but too often for guaranteed services. The aim of this paper is to present a pseudorange nominal measurement model that characterizes the pseudorange measurement error distribution at nominal conditions, that is, with the errors due to any GNSS segment within their specifications and the magnitude of other external error sources within its typical values. Errors resulting from failure of any segment are excluded from this model. In order to be suitable for integrity applications, conservative models are used to bound the large magnitude, low probability errors. The resulting pseudorange error model can be used to define some characteristics of the GNSS application such as the integrity requirements, to identify the threat model and to analyze the integrity risk in the GNSS fault-free case as in [1].

The structure of the paper is the following. First, section II presents the characteristics of the model such as the considered signals, the receiver configurations or the identification of the different sources that cause the pseudorage error. The following sections (sections III to VII) analyze and model each error source individually. Finally, section VIII obtains and studies the final pseudorange error model combining the models of the different error sources calculated in the previous sections.

\section{CHARACTERISTICS OF THE MODEL}

The error model presented in this paper is designed for applications that must meet certain integrity requirements in urban road scenarios. This fact implies certain model characteristics. For instance, integrity requires the use of overbounding techniques and the urban scenario identifies the nature of some error sources. The tracked signals and the receiver configuration also have an important impact on the properties of the error model. This section studies these aspects concerning the error model characteristics.

\section{A. Overbounding}

The design of integrity driven applications relies on the fact that measured errors under nominal conditions are bounded by the nominal measurement model. Additionally, the error pdf is assumed to follow a zero-mean normal distribution. Nevertheless, the error model is commonly obtained from measured or simulated error databases which pdf are not perfectly described by a pure zero-mean normal function. A direct approximation of the model pdf by a Gaussian function with the variance measured from the real data would not bound 
some errors, especially those of large magnitude that occur with low probability. Overbounding techniques are used instead to obtain a conservative pdf that bounds all the errors. Along this work, all error models are overbounding, zero-mean normal functions defined by their variance, $\mathrm{N}\left(0, \sigma^{2}\right)$.

In this paper, error distributions obtained from error databases will be overbounded with the CDF technique described in [2]. This method requires the original error distribution to be symmetric and unimodal. The overbounding is defined in the CDF domain:

$$
\begin{cases}\phi_{O}(x) \leq \phi_{a}(x) & \forall x \leq 0 \\ \phi_{O}(x) \geq \phi_{a}(x) & \forall x>0\end{cases}
$$

where $o$ is the overbounding CDF (zero-mean normal distribution), $\quad a$ is the original CDF of the error database and $x$ is the error magnitude.

\section{B. Error Sources}

The pseudorange measurement error is the result of several independent error sources acting on GNSS signals processing. In a urban road scenario, they are the pseudorange equivalent error due to the satellite's clock and ephemeris inaccuracies, the ionospheric and tropospheric delays, the thermal noise and interferences at the receiver's input and the multipath. The first ones are common to other applications such as civil aviation where they have been widely studied, and multipath has specific effects in urban environments. Each error source is modeled by an overbounding zero-mean normal distribution characterized by its variance. Consequently, the total pseudorange measurement error model, which is the convolution of all the independent error sources, is another overbounding zero-mean normal distribution which variance is the sum of the variance of all error sources:

$$
\sigma_{P S R}^{2}=\sigma_{\text {noise }}^{2}+\sigma_{\text {iono }}^{2}+\sigma_{\text {tropo }}^{2}+\sigma_{\text {clock \& ephem }}^{2}+\sigma_{\text {multipath }}^{2}
$$

\section{Signals and Receiver Configurations}

The nominal measurement model depends on the GNSS signals used and on the receiver configuration. Current and future GNSS systems and combinations of them likely to be used in urban road applications are analyzed: standalone GPS, standalone Galileo, GPS/Galileo, GPS/SBAS and GPS/Galileo/SBAS.

Standard Positioning Service GPS signals at L1, L2 and L5, and Open Service Galileo signals at E1 and E5 are considered. For each code modulation, a suitable receiver configuration is studied (Table 1). Five receiver configurations are considered. Rcx1 represents a low cost BPSK(1) that only tracks the main lobe, and Rcx2 a high performance one that also processes secondary lobes. With a $20 \mathrm{MHz}$ bandwidth, a receiver type Rcx3 only tracks the main lobe of BPSK(10), reason why even if a narrower E-L spacing is possible, it would not improve significantly the signal tracking. A similar reasoning may be applied to Rcx4, designed to track $\operatorname{BOC}(1,1)$. Rcx 5 is able to track up to the main lobe of the $\operatorname{BOC}(6,1)$ component of TMBOC and CBOC signals. Additionally, the E-L spacing of
Rcx4 and Rcx5 has been set in a suitable point of the autocorrelation function, distant from the nulls that would deteriorate the tracking process.

Table 1. Receiver Configurations

\begin{tabular}{|c|c|c|c|}
\hline $\begin{array}{c}\text { Rex } \\
\text { Type }\end{array}$ & $\begin{array}{c}\text { Code } \\
\text { modulation }\end{array}$ & $\begin{array}{c}\text { Front End } \\
\text { BW (MHz) }\end{array}$ & $\begin{array}{c}\text { E-L spacing } \\
\text { (chips) }\end{array}$ \\
\hline Rcx1 & BPSK(1) & 2 & 1 \\
\hline Rcx2 & BPSK(1) & 16 & 0.1 \\
\hline Rcx3 & BPSK(10) & 20 & 0.25 \\
\hline Rcx4 & BOC(1,1) & 4 & 0.2 \\
\hline Rcx5 & TMBOC/CBOC & 14 & 0.1 \\
\hline
\end{tabular}

\section{Noise amplification due to dual frequancy measurements}

Two pseudoranges measured form the same satellite at different frequencies can be combined to remove the ionospheric delay. The counterpart of this procedure is the amplification of those error components that are different at each frequency. The pseudorange equivalent error due to the satellite's clock and ephemeris inaccuracies and the tropospheric delay are identical at both frequencies, so they remain the same. On the other hand, thermal noise, interferences and multipath will be amplified. Assuming that the error component at each frequency is independent:

$$
\begin{aligned}
& \sigma_{\text {iono-free }}^{2}=\left(\frac{f_{A}^{2}}{f_{A}^{2}-f_{B}^{2}}\right)^{2} \cdot \sigma_{A}^{2}+\left(\frac{f_{B}^{2}}{f_{B}^{2}-f_{A}^{2}}\right)^{2} \cdot \sigma_{B}^{2} \\
& \sigma_{\text {iono-free }}^{2}=a^{2} \cdot \sigma_{A}^{2}+b^{2} \cdot \sigma_{B}^{2}
\end{aligned}
$$

where $\sigma_{\text {iono-free }}^{2}$ is the variance of the considered error source (noise plus interferences or multipath) after the combination, $f_{A}$ and $f_{B}$ are the combined carrier frequencies and $\sigma_{A}^{2}$ and $\sigma_{B}^{2}$ are the error variance at each frequency.

The wider is the separation of the frequency combination, the lower is the noise amplification. Table 2 contains the amplification coefficients obtained with the most suitable frequency combinations. Other frequency pairs give much worse results.

Table 2. Noise amplification coefficients in dual frequency measurements

\begin{tabular}{|c|c|c|c|}
\hline $\mathbf{f}_{\mathbf{A}}$ & $\mathbf{f}_{\mathbf{B}}$ & $\mathbf{a}^{2}$ & $\mathbf{b}^{2}$ \\
\hline $\begin{array}{c}\mathrm{E} 1 / \mathrm{L} 1 \\
1575.42 \mathrm{MHz}\end{array}$ & $\begin{array}{c}\mathrm{E} 5 \mathrm{a} / \mathrm{L} 5 \\
1176.45 \mathrm{MHz}\end{array}$ & 5.11 & 1.59 \\
\hline $\begin{array}{c}\mathrm{E} 1 \\
1575.42 \mathrm{MHz}\end{array}$ & $\begin{array}{c}\mathrm{E} 5 \mathrm{~b} \\
1207.14 \mathrm{MHz}\end{array}$ & 5.87 & 2.02 \\
\hline $\begin{array}{c}\mathrm{L} 1 \\
1575.42 \mathrm{MHz}\end{array}$ & $\begin{array}{c}\mathrm{L} 2 \\
1227.6 \mathrm{MHz}\end{array}$ & 6.48 & 2.39 \\
\hline
\end{tabular}




\section{SATELLITE'S CLOCK AND EPHEMERIS EQUIVALENT RANGE ERRORS}

Inaccuracies on the SV clock corrections and ephemeris transmitted in the navigation message yield to pseudorange errors that depend on the GNSS used.

\section{A. Standalone GPS}

The URA (User Range Accuracy) broadcast in the navigation message represents the standard deviation of the error model. Reference [3] proposes a nominal value of URA $=3.9$ meters for the current GPS, which is expected to decrease as the system evolves. The same value of $0.85 \mathrm{~m}$ assumed for the Galileo SISA will be taken for modernized GPS, although the definitions of URA and SISA are different.

$$
\begin{aligned}
& \sigma_{\text {clock \& ephemy, current GPS }}=3.9 \mathrm{~m} \\
& \sigma_{\text {clock \& ephemy, future GPS }}=0.85 \mathrm{~m}
\end{aligned}
$$

\section{B. Standalone Galileo}

The SISA (Signal in Space Accuracy) is the predicted minimum standard deviation of a Gaussian distribution that overbounds the error distribution. A value of $\mathrm{SISA}=0.85$ meters is needed in order to meet the Galileo system RAMS (Reliability, Availability, Maintainability, and Safety) requirements [4]:

$$
\sigma_{\text {clock \& ephemy, Galileo }}=0.85 \mathrm{~m}
$$

\section{C. $S B A S$}

SBAS provides satellite clock and ephemeris corrections denoted fast and long term corrections. The residual error is overbounded by a Gaussian function which standard deviation is defined principally by the parameter UDRE (User Differential Range Error) broadcast by the SBAS. A nominal value of $\mathrm{UDRE}=0.3$ meters is taken.

$$
\sigma_{\text {clock \& ephemy, } S B A S}=0.3 \mathrm{~m}
$$

\section{IONOSPHERIC DELAY}

The velocity of propagation of GNSS signals through the ionosphere depends on their frequency $(f$, in $\mathrm{Hz})$ and the total electron content (TEC, in $\mathrm{e} / \mathrm{m}^{2}$ ) integrated along the ionospheric path from the user to the satellite. The group delay $(I \rho$, in $\mathrm{m})$ first order model is:

$$
I_{\rho} \approx \frac{40.3}{f^{2}} T E C \quad m
$$

The residual error depends on the correction algorithm applied, i.e. dual frequency, GPS, Galileo or SBAS corrections.

\section{A. Dual frequency}

Dual-frequency ionospheric corrections are designed to remove the first order model of the ionospheric delay. Remaining errors due to higher order effects are typically negligible: between 0 and $2 \mathrm{~cm}$ at the zenith for second order effect and between 0 and $2 \mathrm{~mm}$ for the third order ones [5]. Thus, pseudorange errors due to second and higher order ionospheric effects are neglected:

$$
\sigma_{\text {iono, dual frequency }} \cong 0
$$

\section{B. $G P S$}

Single frequency GPS receivers estimate the ionospheric delay ( $T_{\text {iono }}$, in s) using the ionospheric coefficients $\alpha$ and $\beta$ broadcast in the navigation message as the inputs of the correction model described in [6]. The algorithm estimates the ionospheric delay at the user's zenith $\left(T_{\text {iono, vert, }}\right.$ in $\left.\mathrm{s}\right)$ as a function of time and the receiver position, and scales it by the obliquity factor $(F)$ to adjust it according to the satellite's elevation ( $E$, in degrees):

$$
T_{\text {iono }}=F \cdot T_{\text {iono, vert }}
$$

where the obliquity factor expression is:

$$
F=1+16 \cdot\left(0.53-\frac{E}{180}\right)^{3}
$$

Appendix $\mathrm{J}$ of [7] gives the residual error model used in civil aviation for single frequency $\mathrm{L} 1$ receivers. It is an overbounding gaussian function, also suitable for urban integrity applications, which standard deviation, in meters, is:

$$
\sigma_{\text {iono }, G P S}=\max \left\{0.2 \cdot c \cdot T_{\text {iono }}, F_{p p} \cdot \tau_{\text {vert }}\right\}=\max \left\{\Delta_{\text {iono }, 20 \%}, \tau\right\}
$$

where

- $\quad c(\mathrm{~m} / \mathrm{s})$ is the speed of the light in vacuum.

- $T_{\text {iono }}$ is given in seconds

- $F_{p p}=\left[1-\left(\frac{R_{e} \cos E}{R_{e}+h_{I}}\right)^{2}\right]^{-1 / 2}$ is the obliquity factor

- $\quad R_{e}(6378.1363 \mathrm{~km})$ is the semi-major axis of the earth's ellipsoid

- $h_{I}(350 \mathrm{~km})$ is the ionospheric height

- $E$ is the satellite's elevation angle

- $\tau_{\text {vert }}=\left\{\begin{aligned} 9 m, & 0^{\circ} \leq\left|\phi_{m}\right| \leq 20^{\circ} \\ 4.5 m, & 20^{\circ}<\left|\phi_{m}\right| \leq 55^{\circ} \\ 6 m, & 55^{\circ}<\left|\phi_{m}\right|\end{aligned}\right.$ is the minimum vertical delay

- $\phi_{m}$ (deg.) is the geomagnetic latitude

The residual error standard deviation is whether the $20 \%$ of the delay estimated by the correction algorithm $\left(\Delta_{\text {iono, } 20 \%}\right)$ or a minimum value imposed by the model $(\tau)$, whichever is larger. Each term is computed with a different obliquity factor, $F$ and $F_{p p}$, which makes the comparison dependent on the geomagnetic latitude and on the elevation angle. Nevertheless, 
the difference between $F$ and $F_{p p}$ is lower than $3 \%$ for elevation angles above $3^{\circ}$, so $F_{p p}$ may be substituted by $F$ in (11) in order to simplify the equation with a common obliquity factor:

$$
\sigma_{\text {iono,GPS }}=F \cdot \max \left\{0.2 \cdot c \cdot T_{\text {iono, vert }}, \tau_{\text {vert }}\right\} m
$$

Now, only the vertical delay needs to be compared. Considering just the daily maximum ionospheric correction

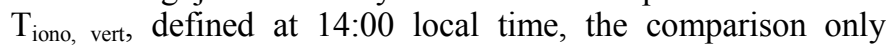
depends on the user's geomagnetic latitude. Numerical values of the daily maximum $T_{\text {iono, vert }}$ have been obtained with a GPS ionospheric correction coefficients database collected since 1994 by the Czech Technical University in Prague. Values have been computed at geomagnetic latitudes ranging from $75^{\circ}$ to $75^{\circ}$ with steps of $1^{\circ}$. The $20 \%$ of the maximum magnitude obtained within each latitude interval defined for $\tau_{\text {vert }}$ is compared against the corresponding $\tau_{\text {vert }}$ in Figure 1. The plot shows that $\tau_{\mathrm{vert}}$ is never reached, so $\tau_{\mathrm{vert}}$ may be considered as a conservative bound which simplifies the model to:

$$
\sigma_{\text {iono,GPS }}=F \cdot \tau_{\text {vert }} \quad m
$$

with $\tau_{\text {vert }}$ as defined in (11).

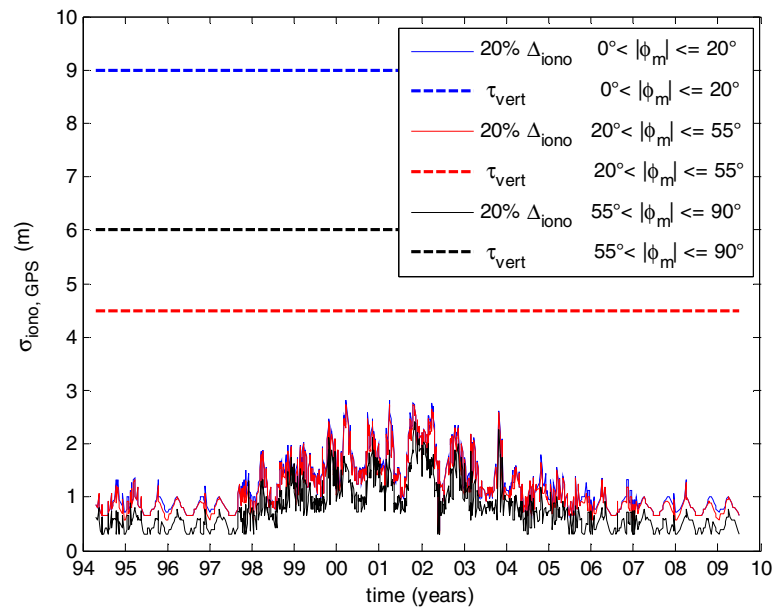

Figure 1. GPS L1 ionospheric residual error model at the zenith Comparison of the $20 \%$ of the daily maximum ionospheric correction and the minimum error imposed by the model.

\section{Galileo}

Galileo single frequency receivers estimate the ionospheric delay using the NeQuick model to compute the TEC integrated along the ionospheric path followed by the signal and applying subsequently the first order model of the ionospheric delay (7) as described in [8]. The input to the NeQuick algorithm is the Effective Ionisation Level $\left(A_{z}\right)$, defined as a function of the ionospheric coefficients broadcast in the Galileo navigation message and the modified dip latitude (MODIP).

The residual error specifications are not to exceed the $30 \%$ of the current ionospheric delay or the equivalent delay due to TEC of $20 \mathrm{TECu}$, whichever is larger [8]:

$$
\sigma_{\text {iono, Galileo }}=\max \left\{0.3 \cdot \Delta_{\text {iono }}, \frac{40.3}{f^{2}} \cdot 20 \cdot 10^{16}\right\} \quad m
$$

Substituting the ionospheric delay by its first order model:

$$
\sigma_{\text {iono, Galileo }}=\frac{40.3}{f^{2}} \cdot 10^{16} \cdot \max \{0.3 \cdot F \cdot V T E C, 20\} \quad m
$$

where VTEC is the vertical TEC and $F$ is the obliquity factor defined in (10). [9].

The residual error model has been assessed and validated in

Numerical values of (15) have been obtained with the International GNSS Service (IGS) VTEC database [10], which provides updated worldwide VTEC maps every 2 hours since 1998. First, the data grid is transformed to convert the geographic latitude into geomagnetic latitude, which is more suitable to describe the ionospheric delay. The conversion, which depends on the latitude ( ) and the longitude $(\lambda)$, limits the final $\mathrm{m}$ to $\pm 75^{\circ}$.

$$
\phi_{m}=\phi+11.6^{\circ} \cdot \cos \left(\lambda-291^{\circ}\right)
$$

The residual error model has been obtained for different percentiles of the VTEC database applying equation (15). The most appropriate percentile curve will be chosen according to the integrity requirements of the given application. As in the GPS model, the model is a function of the absolute value of the geomagnetic latitude because the results at the north and south hemispheres are similar.

Figure 2 compares the GPS and Galileo ionospheric residual error models proposed in this section. Note that they have been obtained differently: the GPS model is the one used in civil aviation, the Galileo one has been obtained from a VTEC database of the last 12 years.

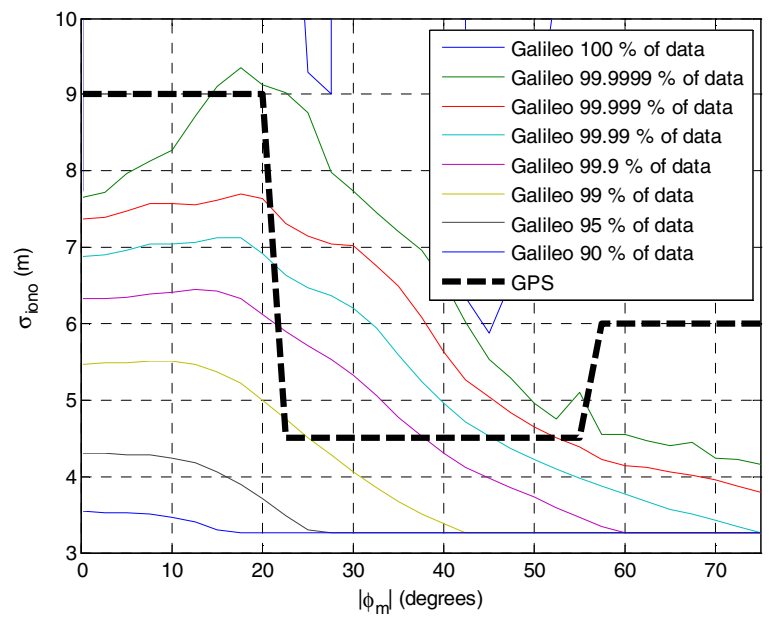

Figure 2. Galileo and GPS ionospheric residual error model

\section{D. $S B A S$}

SBAS broadcast a regional grid of ionospheric corrections and the standard deviation of its residual error at the zenith, GIVE (Grid Ionospheric Vertical Error). Its nominal value 
obtained with a SBAS simulator is GIVE $=0.46$ meters, which is similar to the SBAS vertical ionospheric residual error of 0.5 meters given in [5]. Generally the user is not exactly situated at one of the grid points and computes the ionospheric correction and its associated vertical residual error (UIVE, User Ionospheric Vertical Error) interpolating the grid points closest his position. Assuming all grid points have the same GIVE, the nominal one, the resulting UIVE will be equal to the GIVE. Finally, the nominal error model is the UIVE multiplied by the obliquity factor defined in (10):

$$
\sigma_{\text {iono, } S B A S}=F \cdot \sigma_{U I V E} \quad m
$$

where

$$
\sigma_{U I V E}=0.46 \mathrm{~m}
$$

\section{TROPOSPHERIC DELAY}

Tropospheric delay is caused by tropospheric refraction, which is a local phenomena. Consequently, corrections are not provided by any GNSS and it is the user who directly calculates them with a model stored in the receiver, generally without any other input than the current estimated position. Thus, the residual error only depends on the correction model applied by the user.

The correction algorithm currently used in civil aviation GPS/WAAS receivers provides one the lowest residual errors among the models designed for GNSS applications [11]. Appendix A of [7] describes the algorithm and the residual error model considered in civil aviation:

$$
\sigma_{\text {tropo }}=m(E) \cdot \sigma_{T V E} \quad m
$$

where

$$
\begin{aligned}
& \sigma_{T V E}=0.12 m \\
& m(E)=\frac{1.001}{\sqrt{0.002001+\sin ^{2} E}} \cdot\left(1+0.015 \cdot\left(\max \left[0,4^{\circ}-E\right]\right)^{2}\right) \quad \text { for } E>2^{\circ}
\end{aligned}
$$

Figure 3 represents the tropospheric error model.

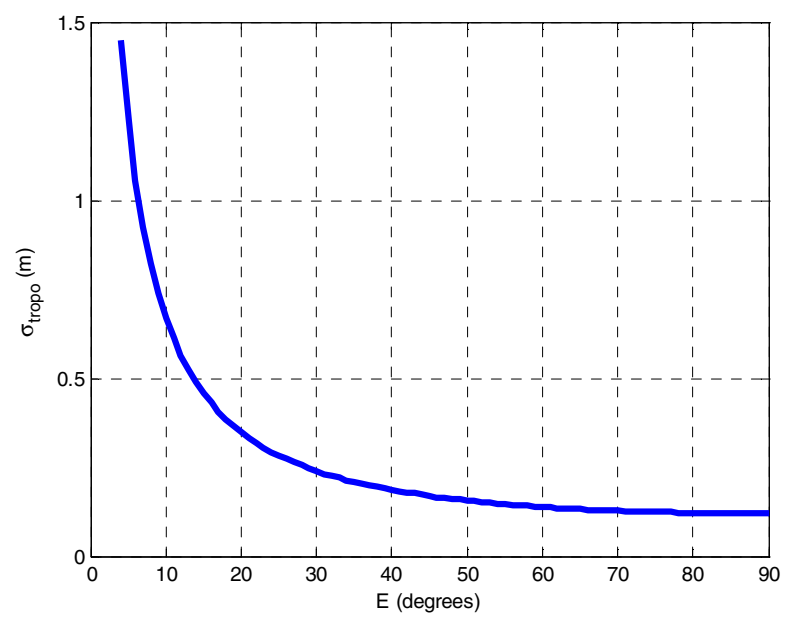

Figure 3. Tropospheric residual error considered in civil aviation.

\section{NOISE AND INTERFERENCIES}

Code tracking error due to thermal noise at the receiver's front-end input depends on the signal modulation and on the receiver design. It has been widely studied and its error model is well known. For Early Minus Late Power (EMLP) discriminators, the variance is given by [12]:

$$
\begin{aligned}
\sigma_{E M L P}^{2}= & \frac{B_{L}\left(1-0.5 \cdot B_{L} T_{I}\right) \int_{-B / 2}^{B / 2} G_{s}(f) \sin ^{2}(\pi f d) \cdot d f}{\frac{C_{s}}{N_{0}}\left(\int_{-B / 2}^{B / 2} 2 \pi \cdot f \cdot G_{s}(f) \sin (\pi f d) \cdot d f\right)^{2}} . \\
& \left(\begin{array}{l}
1+\frac{\int_{-B / 2}^{B / 2} G_{s}(f) \cos ^{2}(\pi f d) \cdot d f}{\frac{C_{s}}{N_{0}} T_{I}\left(\int_{-B / 2}^{B / 2} G_{s}(f) \cos (\pi f d) \cdot d f\right)^{2}}
\end{array}\right) .
\end{aligned}
$$

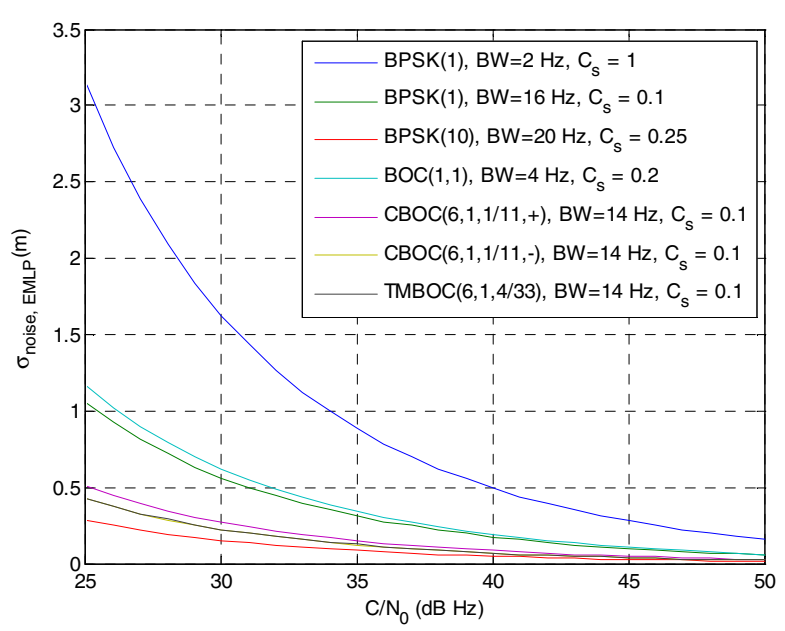

Figure 4. Error due to thermal noise. EMLP discriminator and $\mathrm{T}_{\mathrm{l}}=20 \mathrm{~ms}$ The variance for Dot Product (DP) discriminators is [13]:

$$
\sigma_{D P}^{2}=\frac{B_{L}\left(1-0.5 \cdot B_{L} T_{I}\right) \int_{-B / 2}^{B / 2} G_{s}(f) \sin ^{2}(\pi f d) \cdot d f}{\frac{C_{s}}{N_{0}}\left(\int_{-B / 2}^{B / 2} 2 \pi \cdot f \cdot G_{s}(f) \sin (\pi f d) \cdot d f\right)^{2}} .
$$




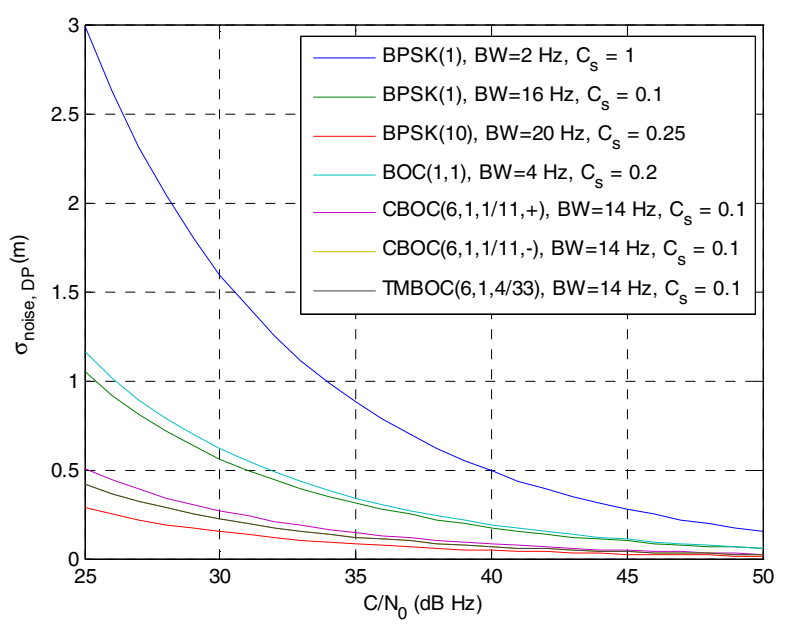

Figure 5. Error due to thermal noise. DP discriminator and $\mathrm{T}_{\mathrm{I}}=20 \mathrm{~ms}$

The effect of interferences can be simulated with the thermal noise obtained by setting the C/No as the PLL loss-oflock threshold.

\section{MULTIPATH}

Multipath effects on pseudorange measurements, especially important in urban scenarios, depend on the user's local environment, so they cannot be mitigated by correction parameters broadcast by any GNSS or SBAS. It is the user that should be provided with a model to bound the possible error due to multipath. This section proposes a multipath error model suitable for integrity applications assuming that the user does not implement any advanced multipath mitigation technique. It has been calculated via Monte Carlo simulations for several signal modulations and receiver configurations. First, the direct signal and multipath parameters are calculated with a channel model. Then, these data are introduced to a receiver simulator to calculate the error caused in the pseudorange measurement. Finally, the error model is obtained with overbounding techniques.

\section{1) Multipath parameters}

The channel model designed for vehicular GNSS users in urban environments described in [14, 15] has been used to compute time series of signal parameters such as the power, phase and delay of the direct and reflected (multipath) rays. The settings of the simulations are the following. The user circulates at $50 \mathrm{~km} / \mathrm{h}$ along the axis of a 20 meter wide road, with the antenna at 1.5 meters of height. The street model has been set to contain trees and buildings at both sides of the road. The buildings average height is 15 meters. The output sampling frequency is $1 \mathrm{kHz}$, which meets for user speeds up to $90 \mathrm{~km} / \mathrm{h}$ the value of $8 / \lambda$ recommended for urban channels [16]. The data has been computed at different azimuth $\left(0^{\circ}, 90^{\circ}\right)$ and elevation $\left(0^{\circ}, 90^{\circ}\right)$ angles with steps of $10^{\circ}$. Simulations have been performed for L1 and they are assumed to be representative of the multipath behavior at other frequencies.

\section{2) Receiver simuator}

The output of the channel model is processed by a receiver simulator to obtain the pseudorange error. The correlator outputs are computed using the direct signal and multipath parameters provided by the channel model with the following model:

$$
\begin{aligned}
& I_{P}=\sum_{i=1}^{N} A_{i} \cdot \widetilde{K}_{c}\left(\varepsilon_{\tau}+\Delta \tau_{i}\right) \cdot \cos \left(\varepsilon_{\theta}+\Delta \theta_{i}\right) \\
& Q_{P}=\sum_{i=1}^{N} A_{i} \cdot \widetilde{K}_{c}\left(\varepsilon_{\tau}+\Delta \tau_{i}\right) \cdot \sin \left(\varepsilon_{\theta}+\Delta \theta_{i}\right) \\
& I_{E}=\sum_{i=1}^{N} A_{i} \cdot \widetilde{K}_{c}\left(\varepsilon_{\tau}+\Delta \tau_{i}+C_{s} / 2\right) \cdot \cos \left(\varepsilon_{\theta}+\Delta \theta_{i}\right) \\
& Q_{E}=\sum_{i=1}^{N} A_{i} \cdot \widetilde{K}_{c}\left(\varepsilon_{\tau}+\Delta \tau_{i}+C_{s} / 2\right) \cdot \sin \left(\varepsilon_{\theta}+\Delta \theta_{i}\right) \\
& I_{L}=\sum_{i=1}^{N} A_{i} \cdot \widetilde{K}_{c}\left(\varepsilon_{\tau}+\Delta \tau_{i}-C_{s} / 2\right) \cdot \cos \left(\varepsilon_{\theta}+\Delta \theta_{i}\right) \\
& Q_{L}=\sum_{i=1}^{N} A_{i} \cdot \widetilde{K}_{c}\left(\varepsilon_{\tau}+\Delta \tau_{i}-C_{s} / 2\right) \cdot \sin \left(\varepsilon_{\theta}+\Delta \theta_{i}\right)
\end{aligned}
$$

where

- $\quad N$ is the total number of LOS and multipath rays

- $\widetilde{K}_{c}$ is the filtered PRN code autocorrelation function, i.e., $\widetilde{K}_{c}(\tau)=\left\{h_{\text {filter }} \otimes K_{c}\right\}(\tau)$

- $C_{s}$ is the Early-Late spacing (chips)

- $\varepsilon_{\tau}=\tau_{1}-\hat{\tau}$, is the DLL synchronization error with regard to the LOS ray

- $\Delta \tau_{i}=\tau_{i}-\tau_{1}$, is the delay of the $i^{\text {th }}$ ray with regard to the LOS ray

- $\varepsilon_{\theta}=\theta_{1}-\hat{\theta}$, is the PLL synchronization error with regard to the LOS ray

- $\Delta \theta_{i}=\theta_{i}-\theta_{1}$ is the phase difference of the $i^{t h}$ ray with regard to the LOS ray

This model considers that the signal parameters (power, phase and delay of each ray) remain constant during the integration time. It is an acceptable assumption because, as seen previously, a $1 \mathrm{~ms}$ sampling period provides an adequate resolution of urban channels for user speeds up to $90 \mathrm{~km} / \mathrm{h}$.

The model also uses the autocorrelation function $\left(K_{c}\right)$ of a whole PRN sequence, although some signals have PRN codes longer than $1 \mathrm{~ms}$. It does not induce a problem neither, because it has been proven during the study that any 1-ms segment of any PRN sequence has autocorrelation characteristics comparable to those of the current L1 C/A code for the needs of our evaluation. 
The characteristics of the implemented phase and code tracking loops are the following. The PLL is a $3^{\text {rd }}$ order Q loop with a bandwidth of $10 \mathrm{~Hz}$, aided by a $2^{\text {nd }}$ order FLL loop with a bandwidth of $15 \mathrm{~Hz}$. The DLL is a $1^{\text {st }}$ order loop with a bandwidth equal to $1 \mathrm{~Hz}$, coupled to the PLL.

At this point, the output of the correlator model has the same sampling frequency as the channel model output $(1 \mathrm{kHz})$. Then, correlator outputs are accumulated along the coherent integration time $T_{I}$ to obtain the correlator output after the Integrate\&Dump. Two values of $\mathrm{T}_{\mathrm{I}}$ are considered, $20 \mathrm{~ms}$ and $100 \mathrm{~ms}$

Afterwards, a lock detector based on PLL and $\mathrm{C} / \mathrm{N}_{0}$ estimators selects the samples that will employ the user. The PLL estimates the carrier phase error [17]:

$$
C 2 \phi_{k}=\frac{N B D_{k}}{N B P_{k}}
$$

where

- $\quad C 2 \phi_{k}$ is the estimated $\cos \left(2 \cdot \varepsilon_{\phi}(k)\right)$

- $N B D_{k}=\left(\sum_{i=1}^{M} I_{i}\right)_{k}^{2}-\left(\sum_{i=1}^{M} Q_{i}\right)_{k}^{2}$ is the Narrow Band Difference

- $N B P_{k}=\left(\sum_{i=1}^{M} I_{i}\right)_{k}^{2}+\left(\sum_{i=1}^{M} Q_{i}\right)_{k}^{2}$ is the Narrow Band Power

- $\mathrm{M}$ is the number of correlator samples used to compute the integrated error, $M=20$ for $T_{I}=20 \mathrm{~ms}$.

Afterwards, the phase estimation may be averaged during a given period, which has been set to $500 \mathrm{~ms}$ in the simulations. Considering as not locked the signals with a carrier phase error higher than $30^{\circ}$ implies a threshold of $\mathrm{C} 2 \mathrm{k}=0.5$. Nevertheless, this PLL cannot detect all possible phase errors, so it has to be used together with a $\mathrm{C} / \mathrm{N}_{0}$ estimator. The following one is used:

$$
C / N_{0}=\frac{\operatorname{mean}\left(I_{p}\right)}{\operatorname{var}\left(I_{p}\right)}
$$

Both mean $\left(I_{p}\right)$ and $\operatorname{var}\left(I_{p}\right)$ are computed from a sequence of the in-phase prompt correlator output. Simulations have been carried out which a sequence length of $500 \mathrm{~ms}$. The PLL threshold $\mathrm{C} 2{ }_{\mathrm{k}}=0.5$ corresponds to a $\mathrm{C} / \mathrm{No}$ of $23 \mathrm{dBHz}$ in the presence of white noise. Then, a C/N0 lock threshold of 23 $\mathrm{dBHz}$ has been taken.

Since the real delay of the signal is known, the computation of the pseudorange error of each sample is straightforward.

\section{3) Overbounding}

Once the error database of the selected samples has been created, it has to be modelled with an overbounding zero-mean normal distribution. As explained previously, the CDF overbounding technique has been used. It has been proven during the study that the original error distributions approximately meet the symmetry and unimodality requirements. The comparison of the simulated and overbounding $\mathrm{CDF}$, pdf and error probability is given in Figure 6 - Figure 8.

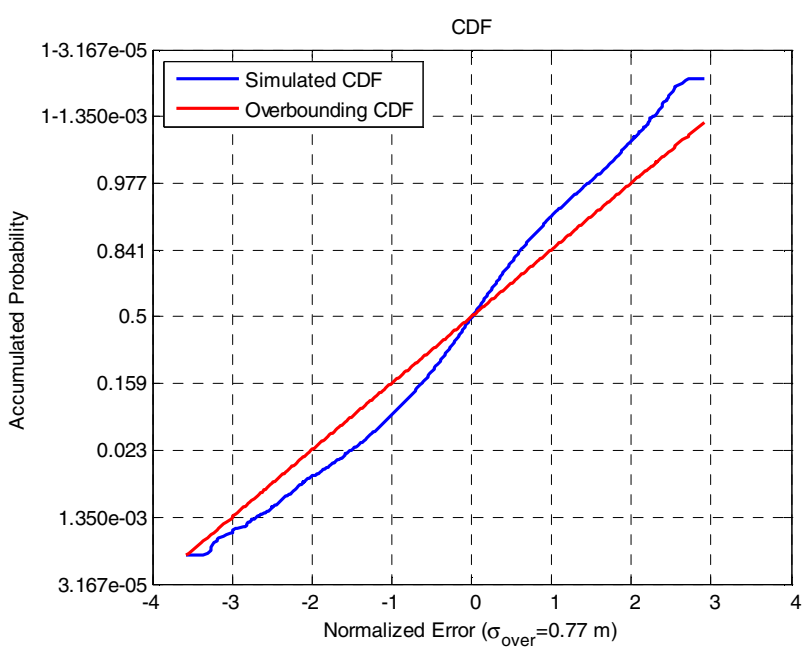

Figure 6. CDF overbounding technique

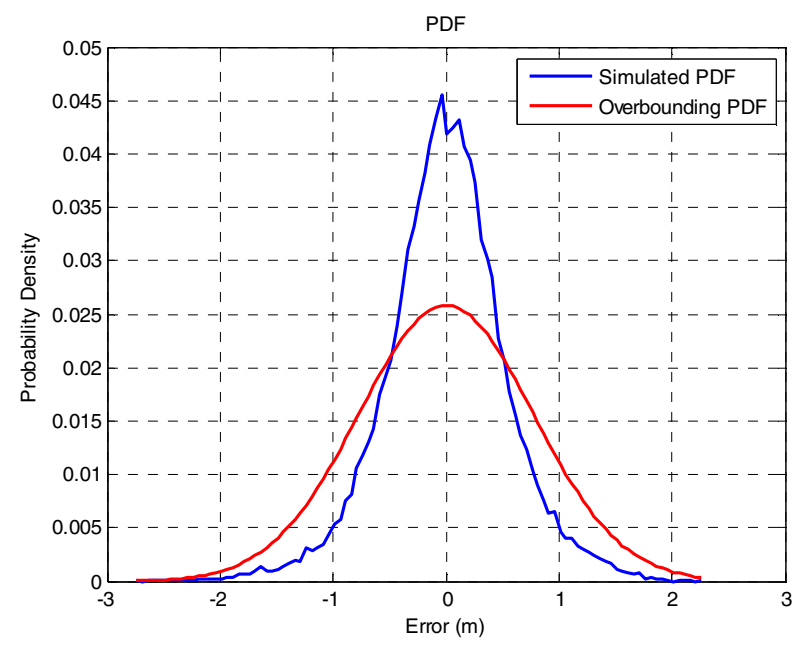

Figure 7. Simulated and overbounding pdf 


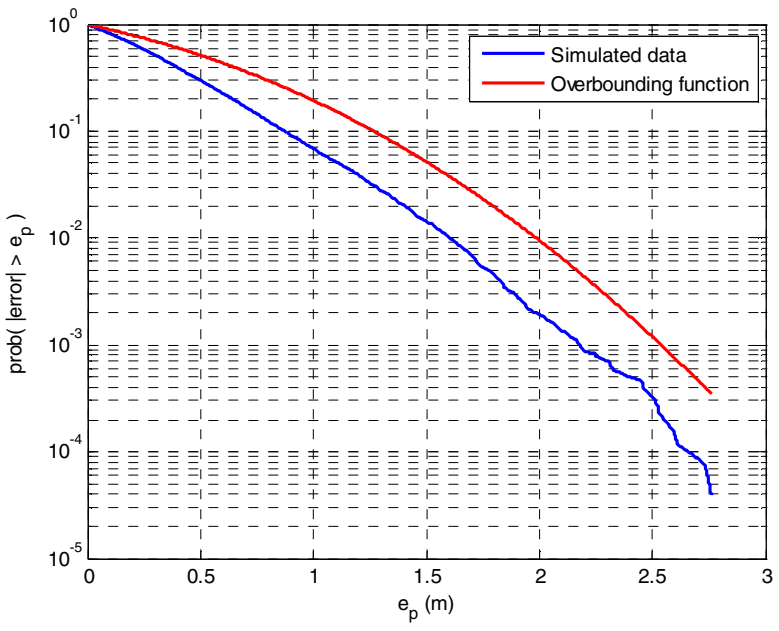

Figure 8. Error probability of simulated and overbounding data

\section{4) Error model}

The multipath error model has been obtained as a function of the elevation angle. The receiver is assumed to work properly and not to introduce additional errors. In addition, the lock detector output is verified by an algorithm that removes isolated large errors. Figure 9 represents the final overbounding error model retained for the different receiver types defined in Table 1 with an integration time of $\mathrm{T}_{\mathrm{I}}=20 \mathrm{~ms}$ and a lock detector averaging period of $500 \mathrm{~ms}$. The simulated signal simulated for $\operatorname{Rex} 5$ is a $\operatorname{CBOC}\left(6,1,1 / 11,{ }^{\prime}-'\right)$. This multipath model is the one kept for the computation of the final pseudorange error.

In order to show the effect of the overbounding process, Figure 10 contains the error standard deviation obtained directly from the simulations (before overbounding). For validation purposes the number of samples used to compute the model at each elevation angle is given in Figure 11.

Except for low cost $\operatorname{BPSK}(1,1)$ receivers, the error model does not depend on the elevation angle. It is also noted that the overbounding model is mainly an amplified version of the error standard deviation before overbounding. Best performances are achieved with $\mathrm{BOC}(10)$, followed by $\mathrm{MBOC}$ and $\mathrm{BOC}$ receivers.

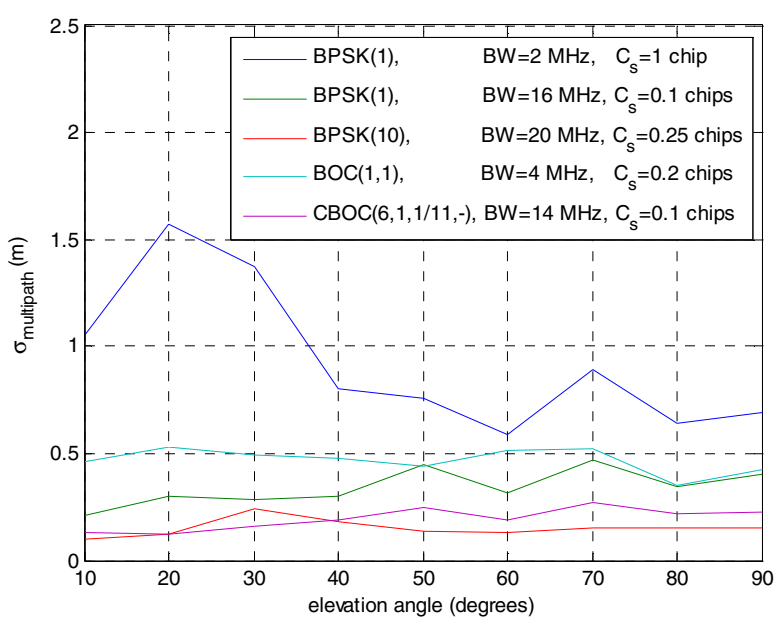

Figure 9. Final multipath overbounding error model $\left(\mathrm{T}_{\mathrm{I}}=20 \mathrm{~ms}\right.$, Lock detector average period $=500 \mathrm{~ms}$ )

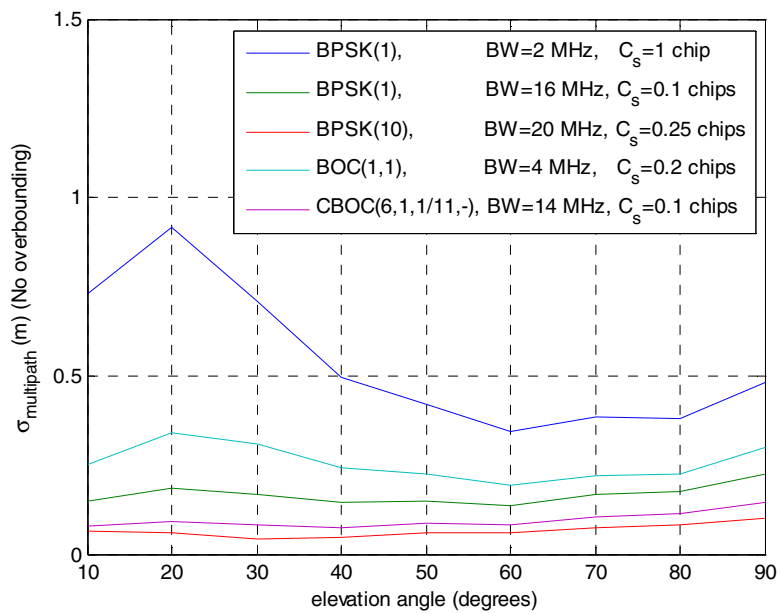

Figure 10. Standard deviation of the multipath error directly obtained from the simulations (No overbounding, $\mathrm{T}_{\mathrm{I}}=20 \mathrm{~ms}$, Lock detector average period $=500 \mathrm{~ms}$ )

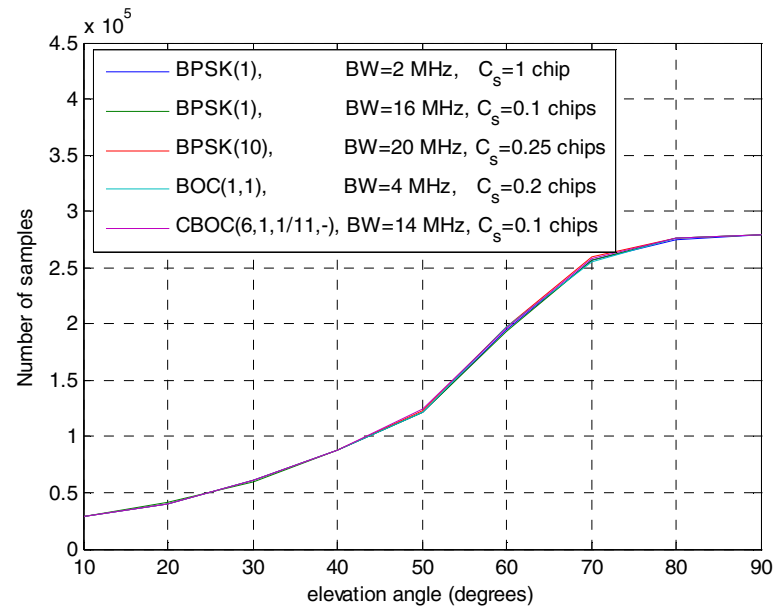

Figure 11. Number of samples used to compute the multipath error model $\left(\mathrm{T}_{\mathrm{I}}=20 \mathrm{~ms}\right.$, Lock detector average period $\left.=500 \mathrm{~ms}\right)$ 


\section{NOMINAL MEASUREMENT MODEL}

The nominal measurement model is the combination of the models calculated in the previous sections for each error source according to the equation (2).

Several examples of pseudorange error model have been plotted in Figure 12 - Figure 16. In all of them the receiver has a DP discriminator and a coherent integration time of $20 \mathrm{~ms}$, the $\mathrm{C} / \mathrm{N}_{0}$ is $40 \mathrm{dBHz}$, the user is at a geomagnetic latitude of $50^{\circ}$ and the equivalent error due to the SV clock and ephemeris inaccuracies is the one corresponding to modernized systems (URA $=\mathrm{SISA}=0.85 \mathrm{~m}$ ). In the MBOC case, simulations have been carried out for $\mathrm{CBOC}(6,1,6 / 11,-)$ signals, even when GPS ionospheric corrections are used. Its performance, although slightly different, is assumed to be representative of that of TMBOC $(6,1,4 / 33)$ and $\operatorname{CBOC}(6,1,1 / 11,+)$ signals.

The main error source of standalone GPS or Galileo is the ionospheric delay (Figure 12 and Figure 13). The effect becomes specially severe at low elevation angles, and could be worse at other latitudes. As the ionospheric delay masks other error sources, there are not significant differences between signals.

Dual frequency receivers eliminate the ionospheric delay at the cost of amplifying the effect of noise and multipath, that become the principal error sources (Figure 14). Because noise and multipath do not depend on the elevation angle, the resulting pseudorange error neither does. In this case, the more robust is a signal against noise and multipath, the lower is the error.

SBAS ionospheric corrections considerably reduce the inospheric residual error of single frequency receivers (Figure 15). The improvement due to the SBAS satellite clock and ephemeris corrections is lower, although it would be more noticeable in the case of current nominal URA (Figure 16). In this case, the error components due to multipath, noise and ionospheric delay present similar magnitudes, so the pseudorange error with SBAS depends on the elevation angle (due to the ionosphere) and on the signal robustness to noise and multipath.

Low cost BPSK(1) receivers, sensible to noise and multipath, offer better performances with SBAS. On the other hand, robust signals like BPSK(10) with low noise and multipath error components, provide better performances with dual frequency receivers than with SBAS, specially at low elevation angles.

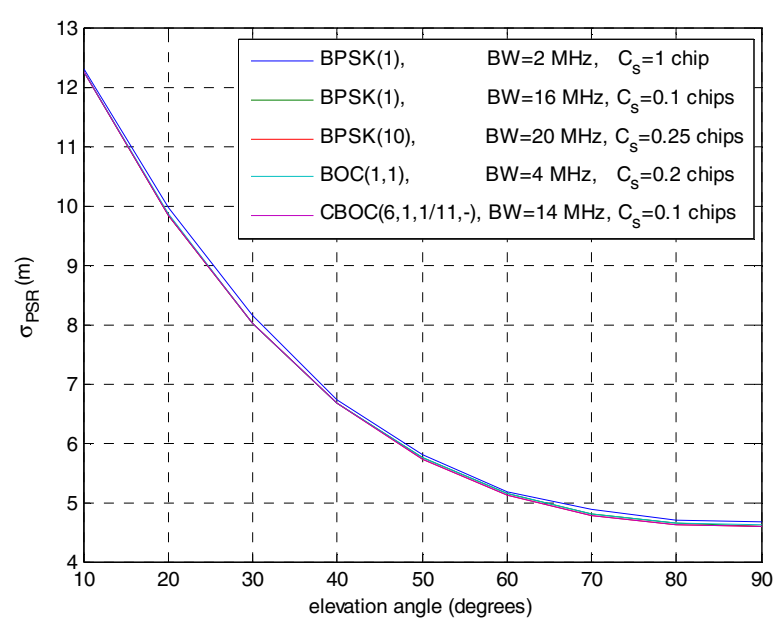

Figure 12. Pseudorange nominal measurement model. Single frequency receiver with GPS ionospheric corrections.

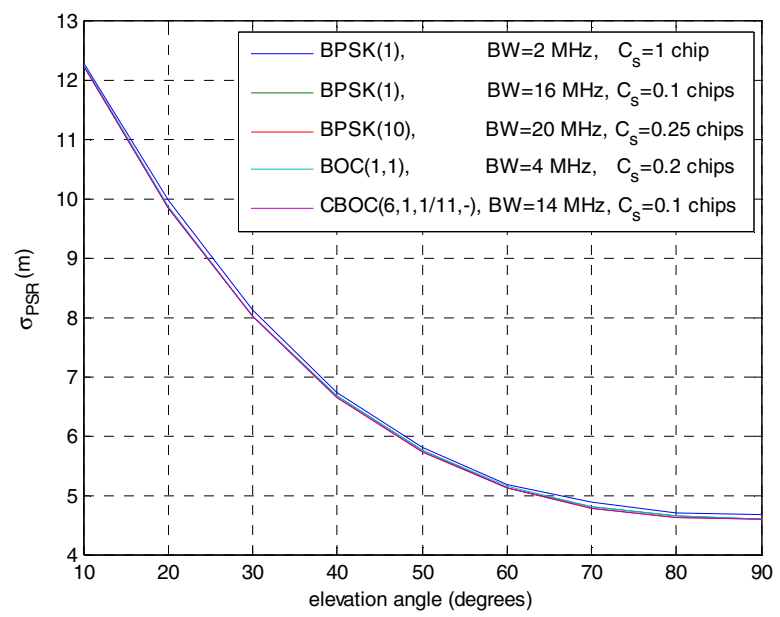

Figure 13. Pseudorange nominal measurement model. Single frequency receiver with Galileo ionospheric corrections.

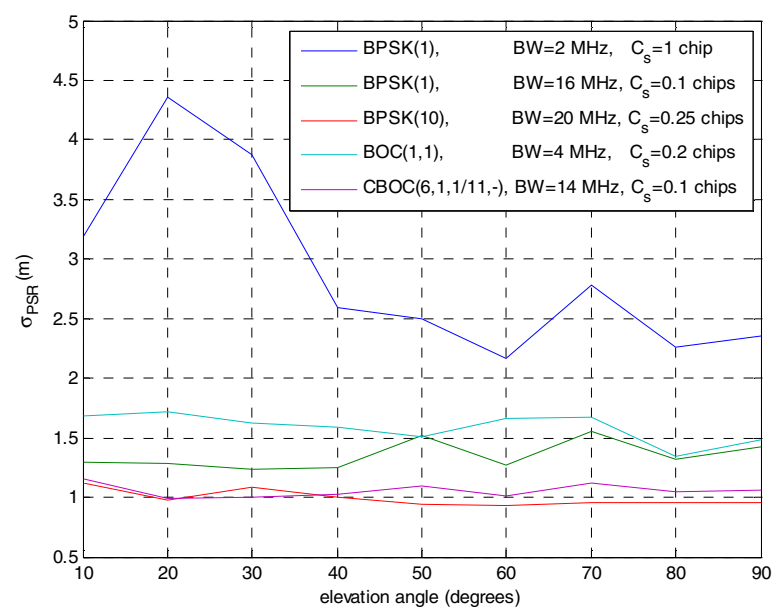

Figure 14. Pseudorange nominal measurement model. Dual frequency receiver. 


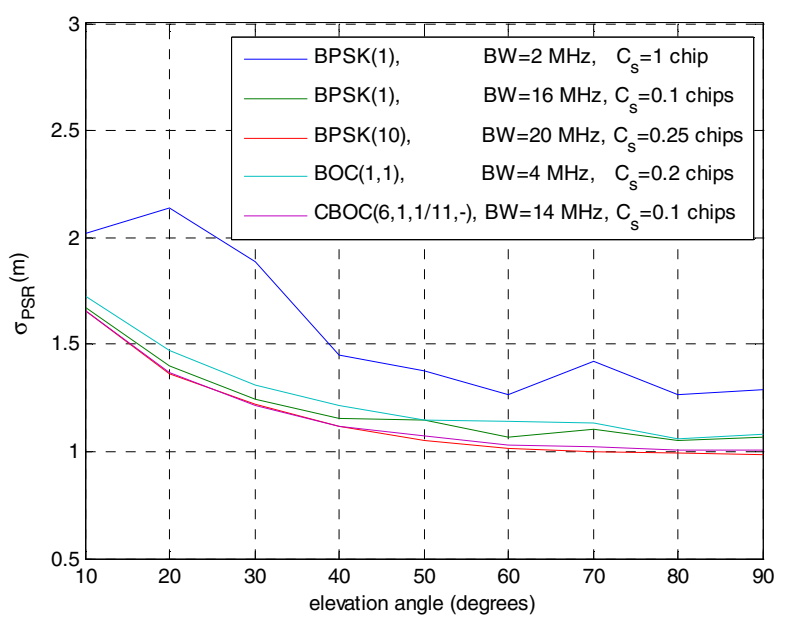

Figure 15. Pseudorange nominal measurement model. Single frequency receiver with SBAS ionospheric corrections.

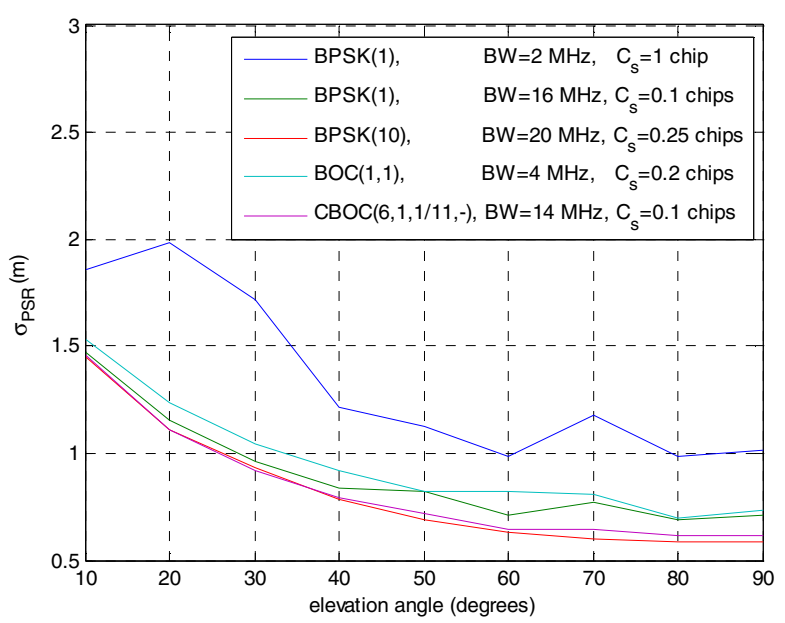

Figure 16. Pseudorange nominal measurement model. Single frequency receiver with SBAS ionospheric, SV clock and ephemeris corrections

\section{CONCLUSIONS}

The pseudorange measurement error for current and future GNSS signals in urban environments has been characterized. The model is an overbounding zero-mean normal distribution suitable to be used in the design of applications with integrity requirements. The different error sources contributing to the pseudorange error have been identified, analyzed and modeled.
Finally, the pseudorange error model has been obtained for different receivers.

\section{REFERENCES}

[1] A. Martineau, "Performance of Receiver Autonomous Integrity Monitoring (RAIM) for Vertically Guided Approaches", PhD thesis, Institute National Polytechnique de Toulouse, 2008

[2] B. DeCleene, "Defining Pseudorange Integrity - Overbounding", ION GPS 2000, 19-22 September 2000

[3] D. Have, "Reference set of parameters for RAIM availability simulations", working paper Sofreavia, 8-9 April, 2003

[4] V. Oehler, F. Luongo, J.P. Boyero, R. Stanford, H.L. Trautenberg, J. Krueger, J. Hahn, F. Amarillo, M. Crisci, B. Schlarmann, J.F. Flamand, "The Galileo Integrity Concept", ION GNSS 2004

[5] M. Mainul-Hoque, M. and N. Jakowski, "Higher order ionospheric effects in precise GNSS positioning"; Journal of Geodesy Vol. 81, pp 259-268

[6] J.A. Klobuchar, "Ionospheric Time-Dealy Algorithm for SingleFrequency GPS Users", IEEE Transactions on Aerospace and Electronic Systems, vol AES-23, no.3, 1987

[7] Radio Technical Commission for Aeronautics (RTCA), "Minimum Operational Performance Standards (MOPS) for Global Positioning System (GPS)/ Wide Area Augmentation System (WAAS) Airborne Equipment", RTCA DO-229D, December 2006

[8] B. Arbesser-Ratsburg, "The Galileo Single Frequency Ionospheric Corection Algorithm", Third European Space Weather Week, 13-17 November, 2006

[9] N.C. Rogers, J.E.N. Field, C. Griffin, P.S. Cannon, M.J. Angling, M. Hollreiser, "An assessment of the Galileo single frequency correction model", Second European Space Weather Week, 14 - 18 November 2005

[10] Hernandez-Pajares, 2008 (VTEC Database) M. Hernández-Pajares, J. M. Juan, J. Sanz, R. Orus, A. Garcia-Rigo, J. Feltens, A. Komjathy, S. C. Schaer, A. Krankowski, "The IGS VTEC maps: a reliable source of ionospheric information since 1998", Journal of Geodesy, 2009, Volume 83, pp. 263-275

[11] J.P. Collins, "Assessment and development of a tropospheric delay Model for Aircraft Users of the Global Positioning System", M.Sc.E. thesis, Department of Geodesy and Geomatics Engineering Technical Report No. 203, University of New Brunswick

[12] Betz J., Kolodziejski K., "Extended Theory of Early-Late Code Tracking for a Band limited GPS Receiver", Journal of the Institute of Navigation, Vol.47, No.3, Fall 2003

[13] Julien O., "Design of Galileo L1F tracking loops", PhD thesis, University of Calgary, Department of Geomatics Engineering, 2005

[14] A. Steingass, A. Lehner, "Measuring the Navigation Multipath Channel - A Statistical Analysis", ION GNSS 17th International Technical Meeting of the Satellite Division, 21-24 September 2004

[15] A. Lehner, A. Steingass, "A Novel Channel Model for Land Mobile Satellite Naviagtion", ION GNSS 18th International Technical Meeting of the Satellite Division, 13-16 Sept. 2005

[16] F. Pérez Fontàn, M. Vazquez Castro, C. Ejamio Cabado, J. Pita Garcia, E. Kubista, "Statistical modelling of the LMS Channel", IEEE Transactions on Vehicular Technology, Vol.50, No.6, Nov. 2001

[17] B.W. Parkinson, J.J. Spilker, P. Axelrad, P. Enge, "Global Positioning System: Theory and Applications. Volume I", Progress in Astronautics and Aeronautics Volume 163, American Institute of Aeronautics and Astronautics (AIAA) Inc., 1996 